\title{
Neuroprotective Effect of Modified Xijiao Dihuang Decoction against Oxygen-Glucose Deprivation and Reoxygenation-Induced Injury in PC12 Cells: Involvement of TLR4-MyD88/NF- $\kappa$ B Signaling Pathway
}

\author{
Xu Zhang, ${ }^{1,2}$ Xiaojun Fei, ${ }^{1,2}$ Weiwei Tao, ${ }^{3}$ Jingbo Li, ${ }^{1,2}$ Hao Shen, ${ }^{1,2}$ Xuanye Wang, ${ }^{1,2}$ \\ Hongquan Liu, ${ }^{1,2}$ and Yun $X u^{4}$ \\ ${ }^{1}$ Jiangsu Province Academy of Traditional Chinese Medicine, Nanjing 210028, China \\ ${ }^{2}$ Affiliated Hospital of Integrated Traditional Chinese and Western Medicine, Nanjing University of Chinese Medicine, \\ Nanjing 210028, China \\ ${ }^{3}$ Center for Translational Systems Biology and Neuroscience, Key Laboratory of Integrative Medicine for Brain Diseases, \\ Nanjing University of Chinese Medicine, Nanjing 210023, China \\ ${ }^{4}$ Nanjing Drum Tower Hospital, Affiliated Hospital of Nanjing University Medical School, Nanjing 210008, China
}

Correspondence should be addressed to Hongquan Liu; njliuhq@126.com and Yun Xu; 568814074@qq.com

Received 1 June 2017; Revised 1 September 2017; Accepted 14 September 2017; Published 30 October 2017

Academic Editor: Nunziatina De Tommasi

Copyright (C) $2017 \mathrm{Xu}$ Zhang et al. This is an open access article distributed under the Creative Commons Attribution License, which permits unrestricted use, distribution, and reproduction in any medium, provided the original work is properly cited.

\begin{abstract}
Modified Xijiao Dihuang (XJDH) decoction has been shown to exert powerful neuroprotective properties in clinical ischemic stroke treatment. It consists of 4 Chinese herbs: Buffalo Horn, Paeonia suffruticosa Andrews, Rehmannia glutinosa (Gaertn.) DC, and Paeonia lactiflora Pall. In the present study, the neuroprotective effect and specific mechanisms of XJDH in protecting PC12 cells from oxygen-glucose deprivation-induced injury were investigated. It was found that OGD/R significantly decreased the cell viability and lactate dehydrogenase (LDH) activity and increased the release of IL- $1 \beta$, IL- 6 , and TNF- $\alpha$ in PC12 cells, and these effects were suppressed by XJDH and one of its major active constituents, paeoniflorin. Additionally, XJDH inhibited caspase-3 activity and reduced cleaved caspase- 3 level. Mechanistic studies showed that the expressions of TLR4, MyD88, TRAF6, and NF$\kappa \mathrm{B}$ p 65 and phosphorylation of $\mathrm{I} \kappa \mathrm{B} \alpha$ and $\mathrm{p} 65$ were significantly lower in the XJDH-treated group than in the OGD/R control group. Additionally, XJDH reversed the OGD/R-induced increases in p-JNK and p-ERK1/2 expression. These results suggest that XJDH protects PC12 cells from oxygen-glucose deprivation-induced injury, which may be associated with the inhibition of the TLR4MyD88/NF- $\kappa$ B signaling pathway. As an anti-inflammation factor, XJDH might be used as a neuronal protection strategy for the ischemia injury and related diseases.
\end{abstract}

\section{Introduction}

Ischemic stroke, also known as cerebral infarction, is considered as one of the leading causes of adult mortality and disability worldwide [1]. About $87 \%$ of stroke cases are due to sudden occlusion of a blood vessel, which may lead to insufficient oxygen and glucose delivery to support cellular homeostasis and then result in cell death $[2,3]$. Therefore, rapid reperfusion in ischemic area for rescuing dying cells is critical in the therapy of cerebral ischemia. However, reperfusion usually causes deterioration of brain injury and a profound inflammatory response [4]. Nowadays few therapeutic options are available for stroke treatment, and thus there is a pressing need for developing new therapeutic strategies.

Although the complex mechanism of cerebra ischemia and reperfusion (I/R) injury has not been exactly demonstrated, the interactions of several pathophysiological processes (i.e., oxygen free radical injury, excitatory amino acid neurotoxicity, and intracellular calcium overload) are shown 
to be involved in stroke progression. Recent evidences have suggested that innate immune and inflammatory reactions are the main cause contributing to the pathophysiology of cerebral I/R injury [4]. Brain ischemia triggers inflammatory responses and produces cytotoxic substances including TNF, IL- $1 \beta$, iNOS, and other proinflammatory mediators, resulting in more neuronal damage [5]. Anti-inflammatory therapy displayed evident neuroprotective effects in ischemia $[6,7]$. Toll-like receptor 4 (TLR4), a germline-encoded pattern recognition receptor expressed by various brain cells, plays an important role in inflammatory responses triggered by $I / R$ injuries [8]. In ischemic/reperfusion condition, TLR4 is activated and leads to recruitment/activation of adaptor protein myeloid differentiation primary response gene (88) (MyD88). Subsequently, the TLR4/MyD88 signaling stimulates TNF receptor-associated factor 6 (TRAF6) and increases the level of nuclear factor kappa B (NF- $\kappa \mathrm{B})$ transcriptional activity and expression of proinflammatory cytokine, thereby triggering inflammatory responses in ischemic brain. Activation of TLR4 also stimulates mitogen-activated protein (MAP) and stress kinases including extracellular-regulated kinase (ERK) $1 / 2$, p38, and Jun kinase (JNK) 1/2, which in turn activates transcription regulators of inflammation. Since inflammatory responses contribute to hypoxic injury and importance of TLR4 in inflammatory mechanisms has been well recognized, blocking TLR4/MyD88 signaling pathway may be a potentially neuroprotective therapeutic strategy for ischemic stroke.

Modified Xijiao Dihuang (XJDH) decoction is derived from a famous ancient Chinese formula originally recorded in "Prescriptions Worth A Thousand Gold," a medical book written by the "Medicinal King" Sun Simiao (around 700 $\mathrm{AD}$ ) [9]. The main herbal ingredients of the formula include Buffalo Horn (Shui Niu Jiao in Chinese), Rehmannia glutinosa (Gaertn.) DC (Sheng Dihuang in Chinese), Paeonia lactiflora Pall. (Shao Yao in Chinese), and Paeonia suffruticosa Andrews ( $\mathrm{Mu}$ Danpi in Chinese). XJDH has been traditionally prescribed for stopping bleeding accompanied with fever, removing toxic substances, and treating spontaneous bleeding, hemoptysis, and nosebleeds [10, 11]. Recently, clinical and preclinical evidences indicated that $\mathrm{XJDH}$ and its components possessed neuroprotective effects against neural damage. In a gerbil model of cerebral ischemia/reperfusion, catalpol, a bioactive constituent of XJDH, was observed to significantly improve the stroke index, increase the activity of SOD, and decrease the brain MDA content [12]. As a main component in Paeonia lactiflora Pall and XJDH, paeoniflorin was able to reduce cerebral infarct and neurological deficit in ischemia-reperfusion injured rats, which at least in part involved the anti-inflammatory properties [13]. In Lu's work, $\mathrm{XJDH}$ was found to improve neurological function deficit in patients with acute cerebral hemorrhage [14]. Although the therapeutic efficiency of $\mathrm{XJDH}$ as a neuroprotective agent is attractive, there were no experimental studies to report the effects of XJDH against ischemic stroke injury for all we know. Moreover, the underlying molecular mechanisms of neuroprotective action of this formula remain unclear.

In the present study, we aim to explore whether $\mathrm{XJDH}$ eases inflammation and neuron damage injury in $\mathrm{PC} 12$ cells after oxygen-glucose deprivation and reoxygenation (OGD/R) and whether the TLR4/MyD88 signaling pathway is related to the potential molecular mechanism.

\section{Materials and Methods}

2.1. Chemicals and Antibodies. L-glutamate and 3-(4,5-dimethylthiazol-2-yl)-2,5-diphenyltetrazolium bromide (MTT) were purchased from Sigma Aldrich (St. Louis, MO, USA). Dulbecco's modified Eagle's medium (DMEM), fetal bovine serum (FBS), and other cell culture reagents were purchased from Gibco-Invitrogen (Carlsbad, CA, USA). Antibodies recognizing TLR4, MyD88, TRAF6, NF- $\kappa$ B, cleaved caspase3 , p-ERK, and p-JNK were supplied by Affinity Biosciences. Secondary antibodies were supplied by Proteintech Group Inc. A lactate dehydrogenase (LDH) ELISA Kit was purchased from Shanghai Enzyme-Linked Biotechnology Co., Ltd.

HPLC-grade acetonitrile and formic acid were purchased from ROE Scientific Inc. (USA). Deionized water was purified using a Milli-Q water purification system from Millipore (Bedford, MA, USA). Reference substances including paeoniflorin, albiflorin, and oxypaeoniflorin were purchased from Must Biological Technology Co. Ltd. (Chengdu, China). The purity of each reference compound was over $98 \%$ by HPLC.

2.2. Preparation of XJDH Decoction. All the crude drugs including $30 \mathrm{~g}$ Buffalo Horn, $24 \mathrm{~g}$ Rehmannia glutinosa (Gaertn.) DC, $12 \mathrm{~g}$ Paeonia lactiflora Pall., and $9 \mathrm{~g}$ Paeonia suffruticosa Andrews were purchased from Jiangsu Provincial Hospital of Traditional Chinese Medicine (Jiangsu Province, China). The voucher sample (number 120320) has been deposited in Affiliated Hospital of Integrated Traditional Chinese and Western Medicine, Nanjing University of Chinese Medicine. Firstly, the mixture of XJDH was soaked in 10 volumes of water for $0.5 \mathrm{~h}$ and refluxed for $2 \mathrm{~h}$. The water extract was filtered, and the residue was refluxed again with 8 volumes of water for $1 \mathrm{~h}$. The combined filtrations obtained were concentrated into the residues in a vacuum evaporator to produce an extract with concentration of $3 \mathrm{~g}$ crude $\mathrm{drug} / \mathrm{mL}$. The XJDH extract was stored at $-80^{\circ} \mathrm{C}$.

2.3. High Performance Liquid Chromatography (HPLC) Analysis and Quantification. For HPLC analysis, XJDH was filtered through a $0.45 \mu \mathrm{m}$ membrane filter before injection. HPLC using Waters 2695 HPLC instrument (Waters Co., Milford, MA, USA) was performed on a SunFire ${ }^{\mathrm{TM}} \mathrm{C} 18$ column $(5 \mu \mathrm{m}$, $250 \mathrm{~mm} \times 4.6 \mathrm{~mm}$ i.d., Waters Co., Milford, MA, USA) with a mobile phase gradient of acetonitrile-water (2\% to $100 \%)$ for $70 \mathrm{~min}$. The injection volume was $10 \mu \mathrm{L}$ of sample and mobile phase flow rate was set at $1 \mathrm{~mL} / \mathrm{min}$ with UV detection at $254 \mathrm{~nm}$. Acquisition and analysis of chromatographic data were performed using Empower software (Waters Co., Milford, USA). Stock solutions of paeoniflorin, albiflorin, and oxypaeoniflorin were prepared for quantification of XJDH. The concentrations of major constituents were determined by regression equations, calculated in the form of $y=a x+b$, where $y$ and $x$ were peak area and contents of the compound, respectively. 
2.4. Cell Culture. PC 12 cells (rat pheochromocytoma cells) were cultured in DMEM medium supplemented with 5\% FBS, $100 \mathrm{U} / \mathrm{mL}$ penicillin, and $100 \mu \mathrm{g} / \mathrm{mL}$ streptomycin at $37^{\circ} \mathrm{C}$ in $5 \% \mathrm{CO}_{2}$ and a saturated humidified incubator. Before the drug treatment, PC12 cells were adhered and grew in the plate for $24 \mathrm{~h}$.

\subsection{Oxygen-Glucose Deprivation and Reoxygenation (OGD/R).} Cells were divided into four groups: vehicle control, OGD/R, $\mathrm{OGD} / \mathrm{R}+\mathrm{XJDH}$, and $\mathrm{OGD} / \mathrm{R}+$ paeoniflorin. For the vehicle control group, PC12 cells were cultured without OGD/R treatment. For the OGD/R group, the original medium was removed, and then cells were rinsed with PBS $(0.01 \mathrm{M}, \mathrm{pH}$ 7.4) twice and exposed to DMEM (glucose free) and cultured in a sealed anaerobic chamber flushed with $5 \% \mathrm{CO}_{2}$ and $95 \% \mathrm{~N}_{2}$ (v/v) for $6 \mathrm{~h}$. XJDH and paeoniflorin were, respectively, added at $30 \mathrm{~min}$ before OGD treatment. Then, a reoxygenation period $(24 \mathrm{~h})$ was begun by rapidly replacing the DMEM (glucose free) with normal medium and conditions.

2.6. Toxicity Assay of XJDH. Cell viabilities of PC12 cells were determined after $24 \mathrm{~h}$ of the treatment with various concentrations of XJDH $(0-2.5 \mathrm{mg} / \mathrm{mL})$ and paeoniflorin $(0-80 \mu \mathrm{M})$ according to the MTT method. The absorbance of the samples was quantified at $490 \mathrm{~nm}$ using spectrophotometer.

2.7. Cell Viability Analysis. PC12 cells were seeded in a density of $5 \times 10^{4}$ cells $/ \mathrm{mL}$ in 96-well plates and cultured for $24 \mathrm{~h}$. Then, the cells were treated with XJDH $(0-2.5 \mathrm{mg} / \mathrm{mL})$ for $30 \mathrm{~min}$ and then subjected to OGD damage. After that, OGD was terminated and the cells were further cultured for $24 \mathrm{~h}$ with $\mathrm{XJDH}$ and paeoniflorin. Cell viability was detected by an MTT assay.

2.8. Lactate Dehydrogenase (LDH) Assay. LDH is a stable cytoplasmic enzyme which catalyzes the interconversion of lactate and pyruvate. When the cell plasma membrane is damaged, intracellular LDH is rapidly released into the culture supernatant. In this work, LDH was detected by a colorimetric assay to investigate cytotoxicity. PC12 cells were treated as described in Section 2.3, and then the cells were lysed using Triton X-100 for $20 \mathrm{~min}$ at $4^{\circ} \mathrm{C}$. After centrifugation, the supernatant was harvested, and $\mathrm{LDH}$ was detected by an LDH assay kit (Shanghai Enzyme-Linked Biotechnology Co., Ltd., Shanghai, China) according to the manufacturer's instructions. The absorbance was detected at $450 \mathrm{~nm}$ and the relative cell viability was calculated as follows: viability $(\%)=\left(\mathrm{OD}_{\text {treatment }}-\mathrm{OD}_{\text {treatment blank }}\right) /\left(\mathrm{OD}_{\text {max. LDH activity }}-\right.$ $\left.\mathrm{OD}_{\text {max. LDH activity blank }}\right) \times 100 \% . \mathrm{OD}_{\text {treatment }}$ is absorbance of drug-treated group; $\mathrm{OD}_{\text {treatment blank }}$ is the background blank absorbance of drug-treated group; $\mathrm{OD}_{\text {max. LDH activity }}$ is absorbance of maximum LDH released group; $\mathrm{OD}_{\text {max. LDH activity blank }}$ is the background blank absorbance of maximum LDH released group.

2.9. Caspase-3 Activity Assay. Caspase-3 activity was detected using caspase-3 ELISA Kit (Shanghai EnzymeLinked Biotechnology Co., Ltd., Shanghai, China). PC12 cells were seeded on $100 \mathrm{~mm}$ dishes for $24 \mathrm{~h}$. After OGD/R treatment as described in Section 2.3, the cells were lysed with lysis buffer (provided by the kit) for $30 \mathrm{~min}$ on ice. Then, cell lysis was centrifuged at $10,000 \mathrm{~g}$ for $15 \mathrm{~min}$ at $4^{\circ} \mathrm{C}$, and the supernatant was harvested. The obtained supernatant was incubated with reaction solution and Ac-LEHD-pNA substrate solution at $37^{\circ} \mathrm{C}$ for $4 \mathrm{~h}$. Finally, the absorbance was detected at $405 \mathrm{~nm}$ and the relative caspase- 3 activities were calculated. Relative caspase-3 activity $(\%)=\left(\mathrm{OD}_{\text {Treatment }}-\right.$ $\left.\mathrm{OD}_{\text {Treatment blank }}\right) /\left(\mathrm{OD}_{\text {Control }}-\mathrm{OD}_{\text {Control blank }}\right) \times 100 \%$.

2.10. Measurement of TNF- $\alpha, I L-1 \beta$, and IL-6 Secretion. The levels of inflammatory mediators such as TNF- $\alpha$, IL- $1 \beta$, and IL-6 in culture medium were determined by ELISA kits (Shanghai Enzyme-Linked Biotechnology Co., Ltd., Shanghai, China) according to the manufacturer's protocols. Concentrations were calculated with reference to the standard curve.

2.11. Western Blot Assay. After treatment as described in Section 2.3, the PC12 cells were lysed in RIPA buffer with cocktail protein inhibitors. Cell lysates were separated by $10-12 \%$ SDS-PAGE and transferred onto a PVDF membrane (Millipore, Billerica, MA, USA). Then, the membranes were soaked with $5 \%$ nonfat milk and individually incubated overnight at $4^{\circ} \mathrm{C}$ with primary antibodies: TLR4, MyD88, TRAF6, NF- $\kappa$ B, cleaved caspase-3, p-ERK, and p-JNKp38. The blots were washed and developed with enhanced chemiluminescent substrate and imaged using ChemiDoc XRS. The optical density (OD) values of the bands were detected by using Carestream Molecular Imaging Software, and statistical data was adjusted to correspond to internal reference expression for eliminating the variations of protein expression (OD value of target protein versus OD of corresponding internal reference).

2.12. Statistical Analysis. All the data were expressed as mean \pm standard deviation (SD). One-way ANOVA was used to determine significant differences among all groups. $P<0.05$ was considered significant.

\section{Results}

3.1. HPLC Analysis of XJDH Decoction. HPLC analysis was performed to determine the content of three major constituents from XJDH decoction. The mobile phase and elution program were optimized. We found that a mobile phase consisting of acetonitrile and $\mathrm{H}_{2} \mathrm{O}$ at optimized elution program can separate paeoniflorin, albiflorin, and oxypaeoniflorin with full peak-to-baseline resolution (Figure 1). Based on UV maximal absorption, we detected paeoniflorin, albiflorin, and oxypaeoniflorin at $254 \mathrm{~nm}$ for quantitative analysis. The contents of paeoniflorin, albiflorin, and oxypaeoniflorin in $\mathrm{XJDH}$ were $0.232 \pm 0.031 \%, 0.276 \pm 0.018 \%$, and $0.019 \pm$ $0.002 \%$, respectively. Linear calibration curve showed good linear regression $\left(r^{2}>0.999\right)$ within test ranges; the LOD $(\mathrm{S} / \mathrm{N}=3)$ and the LOQ $(\mathrm{S} / \mathrm{N}=10)$ were less than 0.5 and $1.0 \mu \mathrm{g}$ for paeoniflorin, albiflorin, and oxypaeoniflorin (Table 1). 


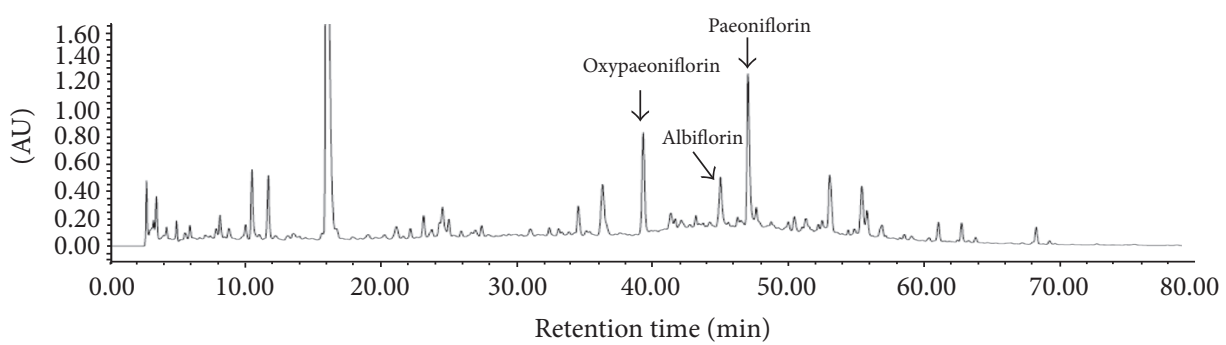

(a)

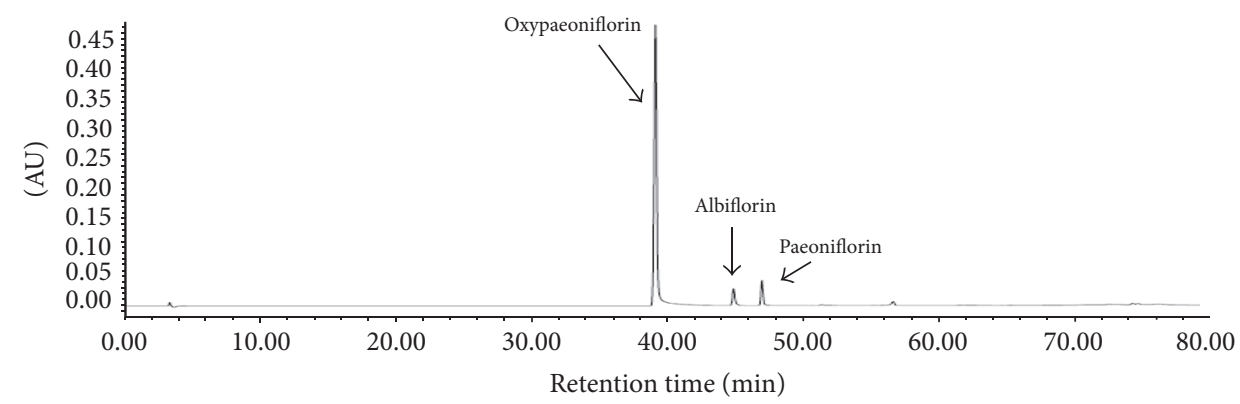

(b)

FIGURE 1: HPLC analysis and quantification of XJDH. HPLC chromatograms of XJDH (a) and oxypaeoniflorin, albiflorin, and paeoniflorin reference (b) obtained using a SunFire ${ }^{\mathrm{TM}} \mathrm{C}_{18}$ column monitored at $254 \mathrm{~nm}$ and eluted with $98 \%$ water to $100 \%$ acetonitrile for $70 \mathrm{~min}$ at a flow rate of $1.0 \mathrm{~mL} / \mathrm{min}$.

TABLE 1: Concentration, calibration curve, regression data, LODs, and LOQs for paeoniflorin, albiflorin, and oxypaeoniflorin by HPLC.

\begin{tabular}{lccccc}
\hline Compounds & Concentration $(\%)$ & Calibration curve & $r^{2}$ & LOD $(\mu \mathrm{g})$ & LOQ $(\mu \mathrm{g})$ \\
\hline Oxypaeoniflorin & $0.019 \pm 0.002$ & $y=1 E+06 x+132000$ & 0.9997 & 0.02 & 0.07 \\
Albiflorin & $0.276 \pm 0.018$ & $y=32176 x+211664$ & 0.9993 & 0.25 & 0.70 \\
Paeoniflorin & $0.232 \pm 0.031$ & $y=110720 x+42996$ & 0.9994 & 0.46 & 0.88 \\
\hline
\end{tabular}

3.2. XJDH Increased Cell Viability after $O G D / R$. First, we examined whether XJDH and its main components paeoniflorin had a toxic effect on cellular viability. PC12 cells were treated with different concentrations of $\mathrm{XJDH}$ and paeoniflorin, and the cell viability was determined using a MTT assay. We found that the cell viabilities of PC12 cells treated with $0.1-1.5 \mathrm{mg} / \mathrm{mL} \mathrm{XJDH}$ were comparable to that of normally cultured cells, indicating that $\mathrm{XJDH}$ did not significantly affect PC12 cell proliferation (data not shown). Similarly, paeoniflorin was not toxic at tested concentrations $(0-80 \mu \mathrm{M})$. Then, we tested whether XJDH and paeoniflorin could protect PC12 cells against OGD/R injury. As presented in Figure 2(a), the viabilities of $\mathrm{PC1} 2$ cells decreased to $43.75 \pm 2.4 \%$ after $6 \mathrm{~h} \mathrm{OGD}$ and $24 \mathrm{~h}$ reperfusion. $\mathrm{XJDH}$ at the concentrations of $0.4,0.2$, and $0.1 \mathrm{mg} / \mathrm{mL}$ notably restored cell viabilities to $91.9 \pm 2.6 \%, 97.3 \pm 3.5 \%$, and $66.9 \pm$ $1.8 \%$. However, XJDH at $2.50 \mathrm{mg} / \mathrm{mL}$ caused an obvious decrease in cell survival. Considering a significant neuroprotective effect of XJDH at $0.2 \mathrm{mg} / \mathrm{mL}$, we chose this concentration to complete the following experiments. Paeoniflorin at concentration of $80 \mu \mathrm{M}$ also significantly attenuated OGD/Rinduced cell death (Figure 2(b)). Release. $\mathrm{LDH}$ is a marker widely used to evaluate the damage and toxicity of cells. To further address the protective activity of XJDH on PC12 cells suffering from OGD/R injury, LDH assay was performed. As shown in Figure 2(c), the relative viability of PC12 cells was significantly suppressed after OGD/ $\mathrm{R}(P<0.01$, compared with vehicle control). When cells were pretreated with XJDH $0.2 \mathrm{mg} / \mathrm{mL}$ and paeoniflorin $(80 \mu \mathrm{M})$, the viability was markedly increased, suggesting an effective neuroprotective effect against $\mathrm{OGD} / \mathrm{R}$ insult.

\subsection{XJDH Reduced Inflammatory Cytokine Level in PC12 Cells} after $O G D / R$. Inflammatory cytokines such as TNF- $\alpha$, IL- $1 \beta$, and IL- 6 play pivotal roles in the pathogenesis of ischemic stroke. In order to investigate the effects of XJDH treatment on the inflammatory response in PC12 cells after OGD/R, we examined the expression levels of TNF- $\alpha$, IL- $1 \beta$, and IL6 in culture medium. As shown in Figure 3, after 6 h OGD exposure, the levels of IL- $1 \beta$, IL- 6 , and TNF- $\alpha$ in the culture medium were significantly elevated approximately 1.45 -fold $(P<0.05), 1.85$-fold $(P<0.05), 1.55$-fold $(P<0.05)$ compared with the control group. When pretreated with $\mathrm{XJDH}$ and paeoniflorin, IL- $1 \beta$, IL- 6 , and TNF- $\alpha$ secretion induced by OGD were remarkably inhibited $(P<0.05$ versus OGD), indicating that XJDH and paeoniflorin could decrease inflammatory response after OGD/R damage. 


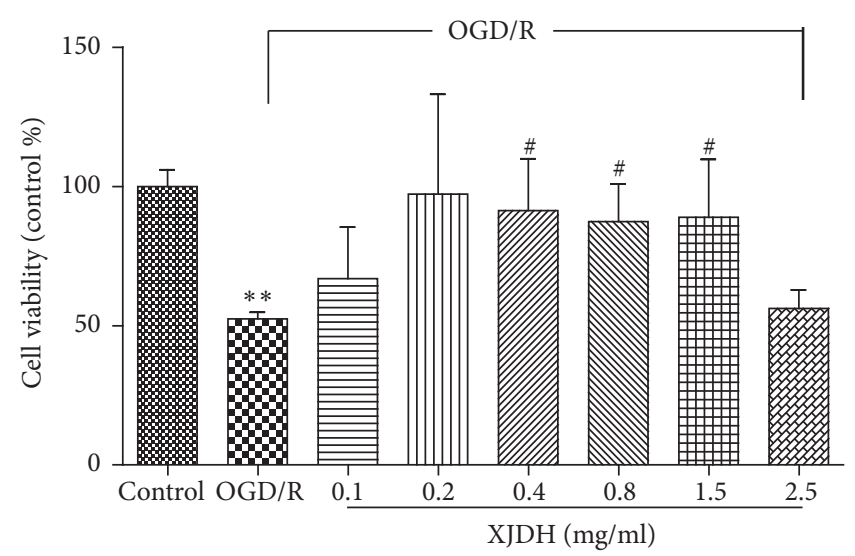

(a)

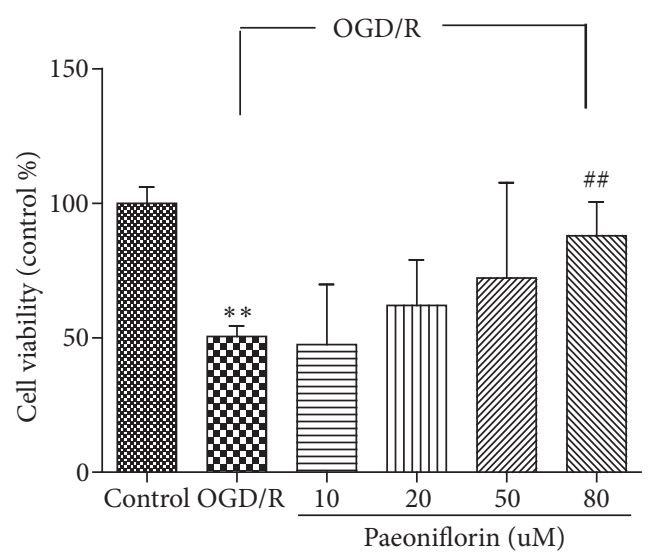

(b)

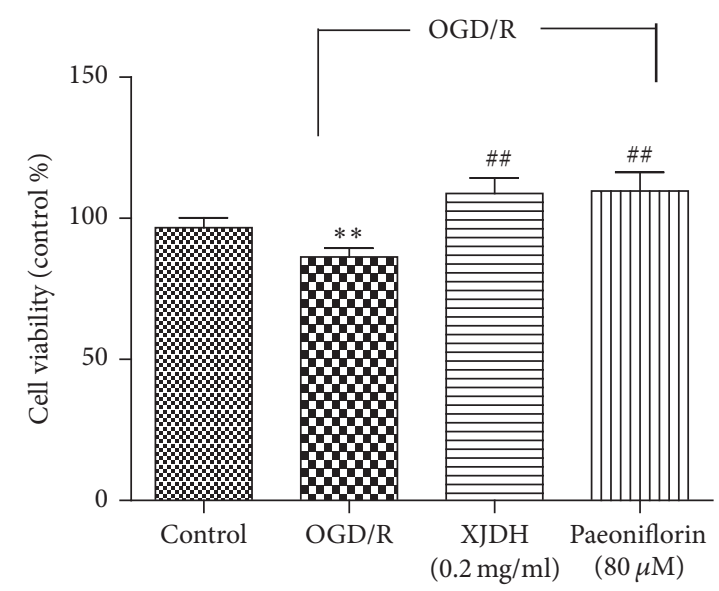

(c)

FIGURE 2: Protective effects of XJDH and paeoniflorin against OGD/R-induced cell death in PC12 cells. Cell viability and toxicity were determined by MTT $(\mathrm{a}, \mathrm{b})$ and LDH assay (c). Cells were pretreated with XJDH and for min, followed by exposure to $6 \mathrm{~h}$ OGD damage and $24 \mathrm{~h}$ reoxygenation. ${ }^{* *} P<0.01$ versus control; ${ }^{\#} P<0.05$ and ${ }^{\# \#} P<0.01$ versus OGD/R-treated cells. All data are represented as the mean \pm SD of three independent experiments.

3.5. XJDH Inhibited the Expressions of TLR4 and Downstream Signaling Ligand MyD88 after OGD/R. Stimulation of TLR4/MyD88 signaling is important for inflammatory responses in ischemic stroke. It is possible that XJDH inhibits the OGD/R-induced inflammation through regulating TLR4 level. To clarify the neuroprotective mechanisms of XJDH, the effects of XJDH on expression of TLR4 and downstream signaling proteins (Figure 4(a)) were investigated using western blotting. To our expectation, $6 \mathrm{~h}$ OGD followed by $24 \mathrm{~h}$ reoxygenation notably induced TLR4 expression and the subsequent signaling effectors activation, reflected by the increased levels of MyD88 and TRAF6, (1.34-, 1.15-fold relative to control, resp.). Western blotting analysis showed that TLR4, MyD88, and TRAF6 protein levels in PC12 cells of the XJDH $(0.2 \mathrm{mg} / \mathrm{mL})$ pretreatment group were significantly decreased compared with those of the OGD/R group. Additionally, TLR4 level and its downstream proteins were also significantly decreased by paeoniflorin (Figure 4(a)). These results suggested that neuroprotective effect of XJDH against OGD/R-induced injury may be mediated by the regulation of TLR4/MyD88 inflammatory signaling pathways.
3.6. XJDH Inhibited NF- $\kappa B$ Activation in PC12 Cells. To further explore mechanisms of XJDH, we investigated effects of $\mathrm{XJDH}$ on $\mathrm{NF}-\kappa \mathrm{B}$ activation in $\mathrm{PC} 12$ cells. Total protein was extracted from PC12 cells $24 \mathrm{~h}$ after the last drug challenge. The expression levels of NF- $\kappa \mathrm{B}$ p 65 and $\mathrm{I} \kappa \mathrm{B} \alpha$ were analyzed by western blot analysis. Our data showed that $\mathrm{OGD} / \mathrm{R}$ stimulation induced the phosphorylation of both p65 and $\mathrm{I} \kappa \mathrm{B} \alpha$ (Figure $4(\mathrm{~b}))$. XJDH $(0.2 \mathrm{mg} / \mathrm{mL})$ attenuated the phosphorylation of $\mathrm{I} \kappa \mathrm{B} \alpha$ and p 65 (Figure $4(\mathrm{~b}))$. The total NF- $\kappa$ B p 65 subunit showed similar patterns as p-p65. These results confirmed that XJDH inhibited TNF- $\alpha$ induced NF$\kappa \mathrm{B}$ activation in $\mathrm{PC} 12$ cells.

3.7. XJDH Had Little Effect on MAPK Pathway in $O G D / R$ Treated PC12 Cells. TLR4 activation may stimulate phosphorylation of MAPK pathway. Hence we investigated whether MAPK signaling participated in the neuroprotective effect of $\mathrm{XJDH}$ on OGD/R injury. As illustrated in Figures 5(a) and 5(b), PC12 cells exposed to OGD/R showed an increase in the phosphorylation of ERK1/2 and JNK (both at $P<0.05$ ). 

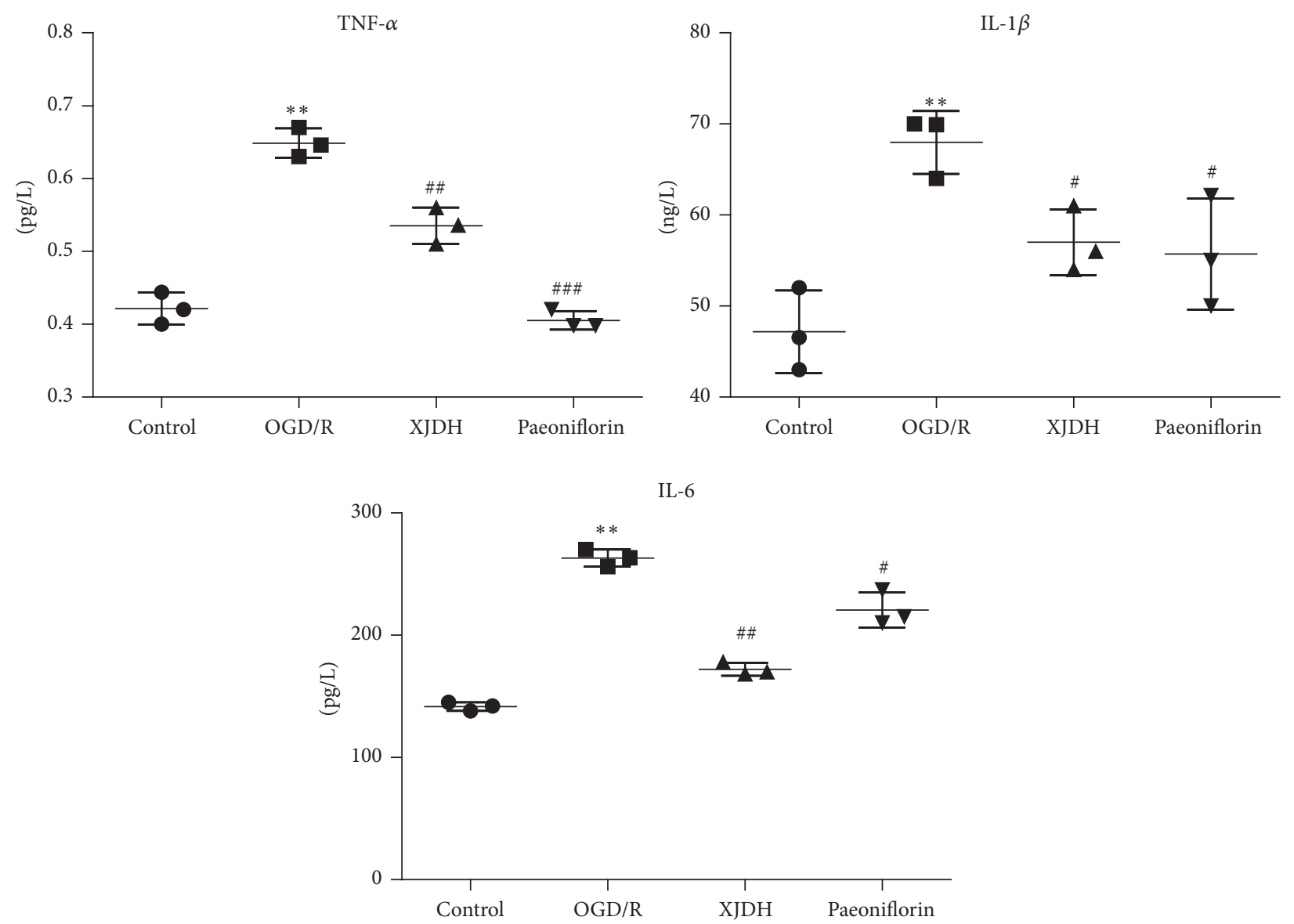

FIGURE 3: Effects of XJDH and paeoniflorin on IL-1 $\beta$, IL-6, and TNF- $\alpha$ secretion in OGD/R-treated PC12 cells. ${ }^{* *} P<0.01$ versus control; ${ }^{\#} P<0.05,{ }^{\# \#} P<0.01$, and ${ }^{\# \# \#} P<0.001$ versus OGD/R-treated cells. All data are represented as the mean \pm SD of three independent experiments.

The increased expression levels of p-ERK1/2 and p-JNK induced by $\mathrm{OGD} / \mathrm{R}$ were reduced and pretreated with $0.2 \mathrm{mg} / \mathrm{mL} \mathrm{XJDH}$ or $80 \mu \mathrm{M}$ paeoniflorin. However, there was no significant difference between the OGD/R group and $\mathrm{XJDH}$-treated group. These data suggested that XJDH did not exert its protective effects on PC12 cells through regulating MAPK pathway.

\subsection{XJDH Inhibited Caspase-3 Activity and Cleaved Caspase-} 3 Expression in OGD/R-Induced PC12 Cells. Caspases-3 played essential roles in apoptosis after cerebral ischemia. In this study, we also investigated the mechanisms by which $\mathrm{XJDH}$ and paeoniflorin worked against OGD-induced injury, by analyzing the caspases-3 activity. Figure 6(a) revealed that OGD induced dramatic increase of caspases- 3 activity level compared with vehicle control group. Here, XJDH and paeoniflorin treatment significantly downregulated the caspases-3 activity in PC12 cells. In Figure 6(b), OGD insult induced a significant increase in expression level of the proapoptotic protein cleaved caspase-3, which was inhibited by XJDH and paeoniflorin. These data indicated that caspase3 apoptosis signal pathway may contribute to the neuroprotective action of XJDH in PC12 cells.

\section{Discussion}

Stroke is one of the leading causes of morbidity and mortality in humans which arises from thrombosis, embolism, or systemic hypoperfusion. Many drugs show neuroprotective effects for stroke treatment, including NMDA receptor antagonist, calcium antagonists, and pituitary adenylate cyclaseactivating polypeptide (PACAP) $[15,16]$. Nowadays, with advantage of minimal side effects, more and more attention has been focused on constituents from natural medicines or traditional Chinese medicine (TCM) preparations in ischemic stroke therapy [17]. In the present study, we demonstrated that XJDH protected PC12 cells from OGD/R-induced cell damage. OGD/R model is considered as a highly reproducible and appropriate in vitro model of ischemic stroke. By developing OGD/R model in PC12 cells, we found that XJDH administered prior to the insult could prevent the OGD/Rinduced cell viability decrease and LDH release. Mechanistic studies showed that the neuroprotective effect of XJDH was performed by inhibiting protein expression of TLR4, MyD88, and TRAF6, NF- $\kappa$ B activation, secretion of TNF- $\alpha$, IL- 6 , and IL-1 $\beta$, and caspase- 3 activity. Activation of TLR4 also stimulates mitogen-activated protein and stress kinases which in 

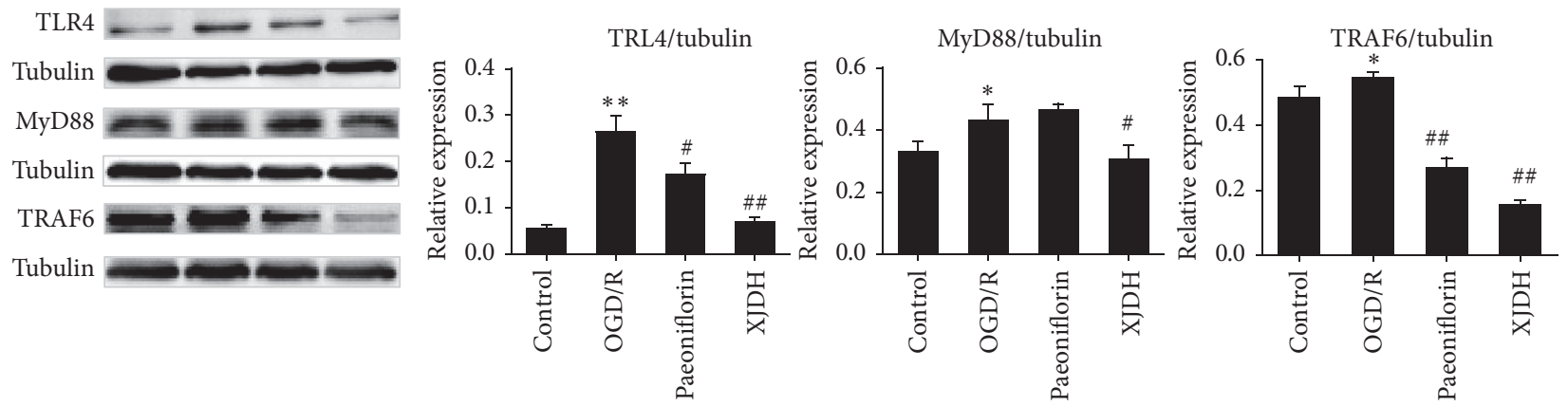

(a)

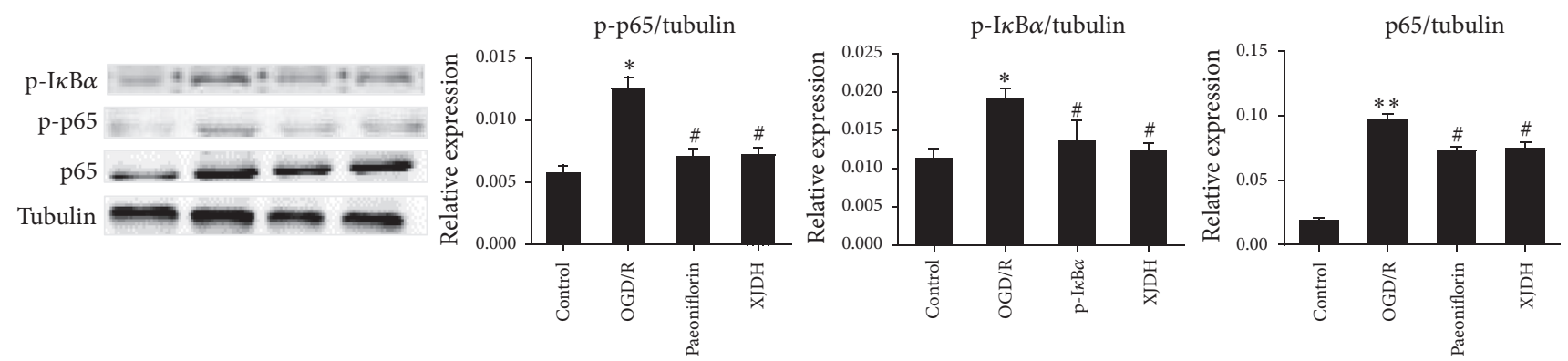

(b)

FIgURE 4: Protective effects of XJDH and paeoniflorin on regulation of proteins in TLR4-MyD88/NF- $\kappa$ B signaling pathway in PC12 cells. Expressions of TLR4, MyD88, and TRAF6 (a) and phosphorylation of $\mathrm{I} \kappa \mathrm{B} \alpha$ and NF- $\kappa \mathrm{B}$ p 65 (b) were detected by western blots, and tubulin was used as a control. Bar graph represents semiquantitative densitometry from western blot analysis. ${ }^{*} P<0.05$ and ${ }^{* *} P<0.01$ versus control; ${ }^{\#} P<0.05$ and ${ }^{\# \#} P<0.01$ versus OGD/R-treated cells.

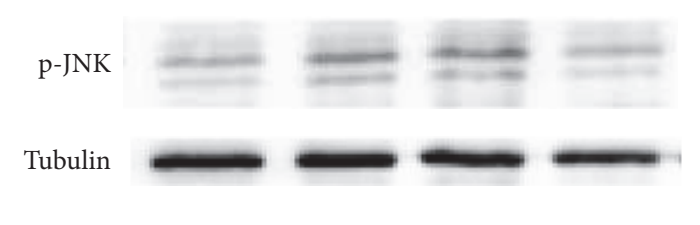

p-JNK

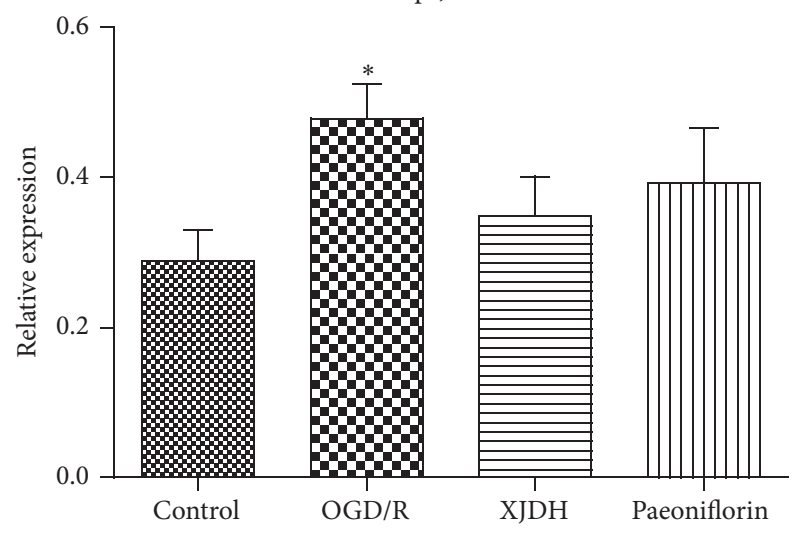

(a)
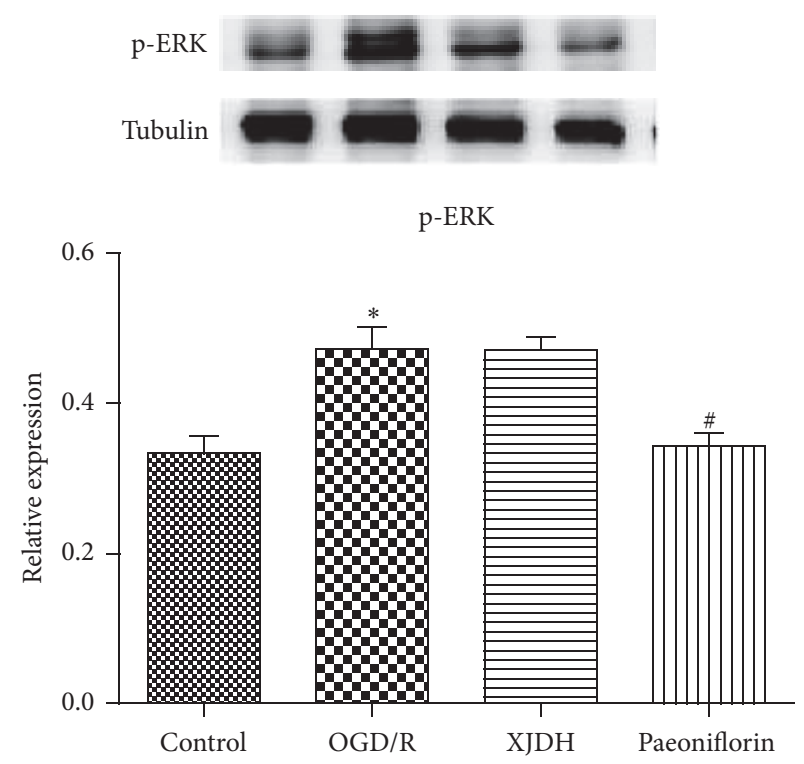

(b)

FIGURE 5: The impact of XJDH and paeoniflorin on protein expression of p-ERK1/2 and p-JNK1/2 in MAPK signaling pathway (a). Bar graph represents semiquantitative densitometry from western blot analysis (b). ${ }^{*} P<0.05$ versus control; ${ }^{\#} P<0.05$ versus OGD/R-treated cells. 


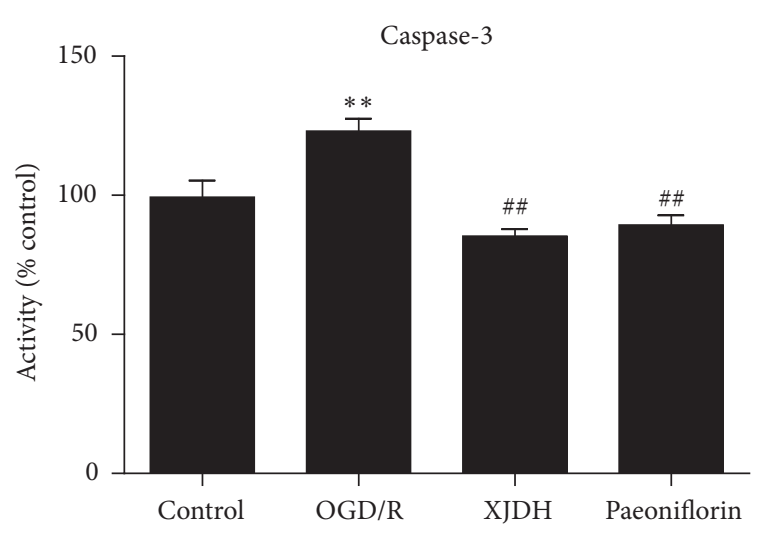

(a)

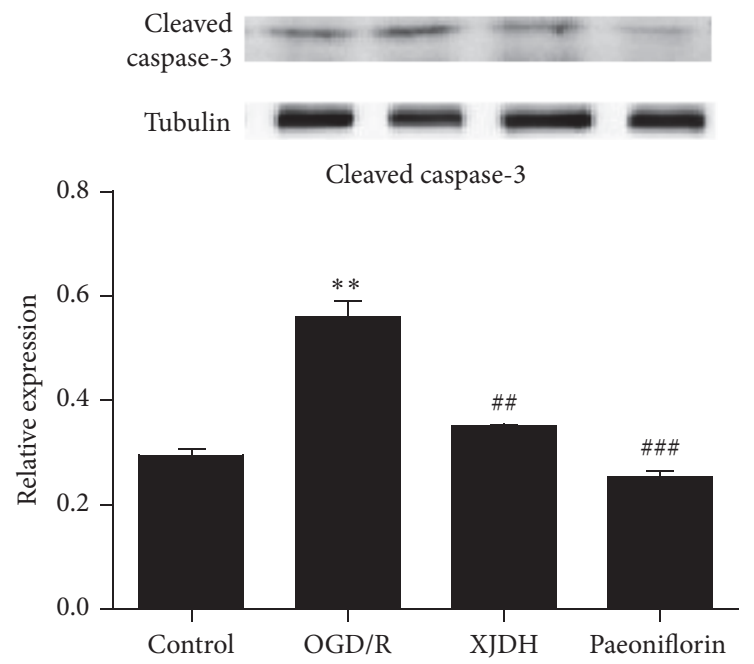

(b)

FIGURE 6: Effects of XJDH and paeoniflorin on caspases-3 activity (a) and cleaved caspase-3 protein expression (b). ${ }^{* *} P<0.01$ versus control; ${ }^{\# \#} P<0.01$ and ${ }^{\# \#} P<0.001$ versus OGD/R-treated cells.

turn activate transcription regulators of inflammation. Therefore, we investigated whether MAPK pathway contributed to the neuroprotective effect of XJDH. The results showed that XJDH has little effect on the increased expression of $\mathrm{p}$ ERK and p-JNK triggered by OGD/R exposure. XJDH is a neuroprotective agent that potentially explains the alleviation and prevention from hypoxia-ischemia-induced injury in neurons.

Inflammatory response is a key factor contributing to ischemia-induced injuries and neuronal apoptosis [4]. Cerebral ischemia triggers inflammatory responses and numerous inflammatory mediators that exacerbate ischemic brain injury are induced at the transcriptional level, including enzymes required for prostaglandin synthesis, cytokines (e.g., IL-6 and TNF- $\alpha$ ), and chemokines [18]. TLR4, an innate and adaptive immune cell receptor of the pathogen recognition receptor family and damage-associated molecular patterns (DAMP) recognition, has increasingly been recognized to be implicated in cerebral I/R injury [19]. Several reports have demonstrated that TLR4 expression was significantly increased in neural tissue after cerebral I/R injury compared to sham groups $[8,20]$. The increase of TLR4 expression would activate TRAF6/NF- $\kappa \mathrm{B}$ signaling by the MyD88. NF- $\kappa \mathrm{B}$, a key transcription factor of inflammatory cascades, therein initiates the transcription of proinflammatory cytokines (e.g., TNF- $\alpha$, IL-1 $\beta$, and IL-6). Data from human studies suggested the levels of TNF- $\alpha$ and IL- 6 were positively correlated with infarct volume and poor clinical outcome [21]. Therefore, regulating TLR4/NF- $\kappa \mathrm{B}$ signaling pathway following cerebral I/R injury is a viable target for the treatment of cerebral I/R injury. Neurons deficient in TLR4 showed increased resistance and less apoptotic cell death when subjected to glucose deprivation in an in vitro model of ischemic conditions [20]. Animal studies also suggested that TLR4 deficiency reduced infarct sizes and improved neurological outcome [22, 23]. Following TLR4 blockade, NF- $\kappa \mathrm{B}$ expression was downregulated compared to cerebral $\mathrm{I} / \mathrm{R}$ injury alone, while the extent of cerebral I/R injury was also reduced [24]. In the present study, our results showed that $\mathrm{XJDH}$ at $0.2 \mathrm{mg} / \mathrm{mL}$ significantly decreased the levels of TNF- $\alpha$, IL- $1 \beta$, and IL- 6 in OGD/R-treated PC12 cells, indicating that $\mathrm{XJDH}$ decreased neuroinflammation after $\mathrm{OGD} / \mathrm{R}$. In addition, $\mathrm{XJDH}$ markedly decreased the expression levels of TLR4, MyD88, TRAF6, and NF- $\kappa$ B p65 and attenuated the phosphorylation of $\mathrm{I} \kappa \mathrm{B} \alpha$ and $\mathrm{p} 65$. These results showed that TLR4-MyD88/NF- $\kappa \mathrm{B}$ signaling pathway may be involved in the neuroprotective action of XJDH.

Activation of TLR4 also stimulates MAPK pathway, which plays a critical role in I/R injury. Reports have shown that pharmacological ERK and JNK blockade could prevent OGD/R-induced inflammatory response and improved the outcome of ischemic brain injury by downregulating apoptosis signaling $[25,26]$. The roles of ERK in the pathophysiology of cerebral ischemia are still controversial. Some reports demonstrated that the phosphorylation of ERK after ischemia might support neuronal damage [27]. In this paper, the effect of XJDH on phosphorylation of ERK and JNK was investigated. Consistent with previous reports, we observed that the levels of p-ERK and p-JNK were persistently increased after OGD/R. The administration of XJDH prevented the increased level of $\mathrm{p}$-ERK and p-JNK to some extent. However, there was no significant difference between the OGD/Rtreated group and XJDH-treated group. These data suggested that MAPK pathway may have little contribution to the pharmacological action of XJDH against OGD/R injury.

Caspase-3, a key mediator of apoptosis, is thought to play a central role in cell apoptosis. Active caspase- 3 can lead to DNA fragmentation and eventually apoptosis [28]. 


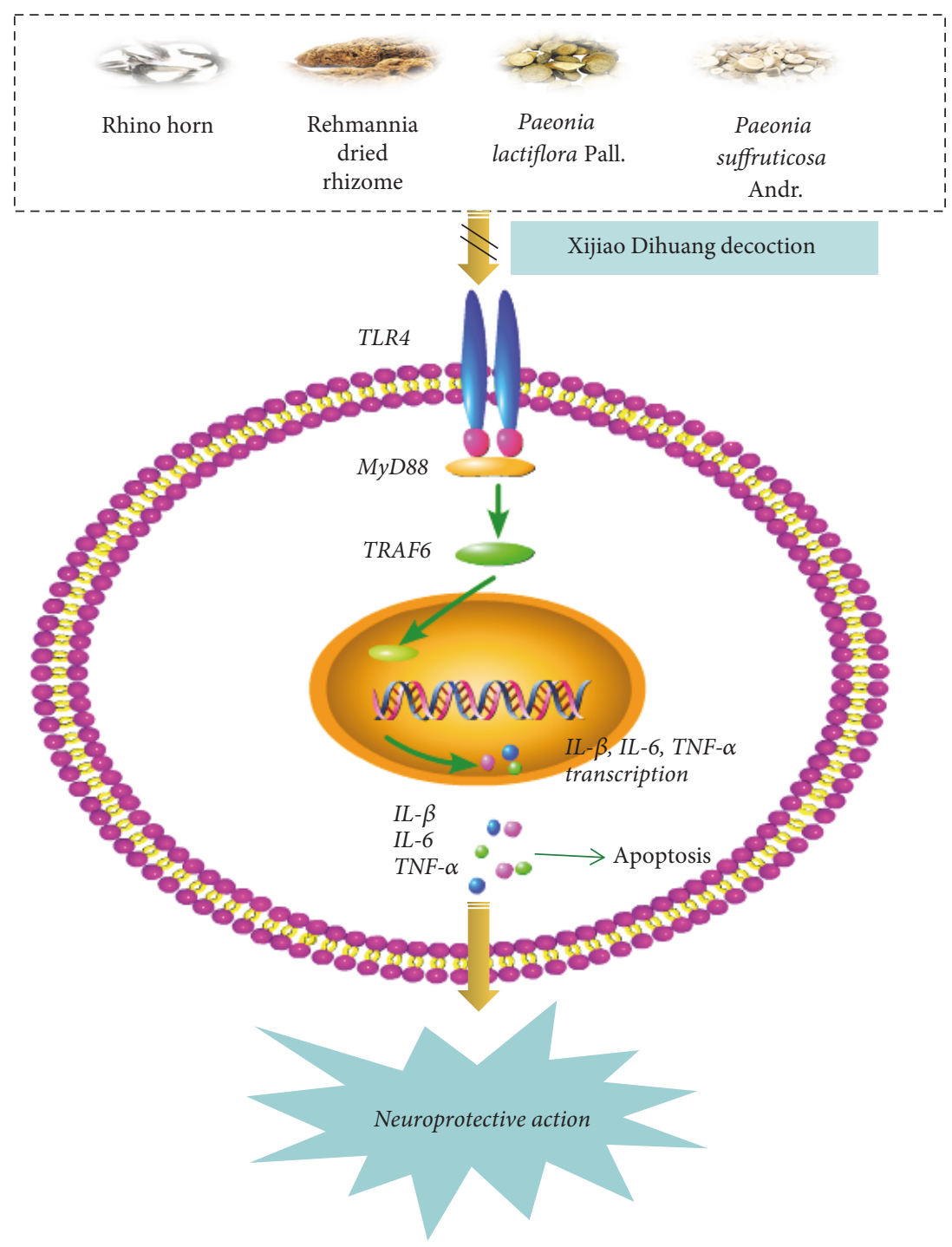

FIGURE 7: Proposed model for the neuroprotective effects of XJDH in OGD/R injury. XJDH inhibits TLR4/MyD88/NF- $\kappa$ B signaling to ameliorate neuroinflammation and OGD/R injury.

Studies have showed that activated caspase- 3 cleaves proteins that were essential in maintaining neuronal process integrity [29]. Several hours after hypoxia-ischemia, neurons started to undergo caspase-3-dependent apoptosis [30]. In our work, the effect of XJDH on caspases-3 activity and cleaved caspase3 protein expression were also tested. We found that XJDH significantly decreased caspases-3 activity and the level of cleaved caspases- 3 in PC1 2 cells exposed to OGD/R, suggesting that the action of XJDH against injury by OGD/R could be partly related to its antiapoptotic effects.

To sum up, the study presented here, for the first time, the fact that herbal medicine XJDH ameliorated the OGD/R-induced damage in PC12 cells, which was associated with its inhibition to TLR4-MyD88/NF- $\kappa$ B pathway and the proinflammatory cytokines (Figure 7 ). Based on results, we provide a possible explanation and the opportunity for the evaluation of XJDH in the treatment of brain injury. However, more in-depth mechanisms and clinical studies of $\mathrm{XJDH}$ against ischemic stroke should be further elucidated for its application in the clinical practice.

\section{Conflicts of Interest}

The authors declare no conflicts of interest with the content of the paper.

\section{Authors' Contributions}

$\mathrm{Xu}$ Zhang and Xiaojun Fei performed the majority of the experiments and designed the research and wrote the manuscript. Weiwei Tao, Jingbo Li, Hao Shen, and Xuanye Wang supported several experiments. Hongquan Liu and Yun $\mathrm{Xu}$ designed the research and contributed materials and revised the manuscript. Xu Zhang and Xiaojun Fei contributed equally to this work. 


\section{Acknowledgments}

This study was supported by National Natural Science Foundation of China (no. 81573964) and the Jiangsu Bureau of Traditional Chinese Medicine (no. LZ13075) and was also funded by grants from Six Big Talent Peak Project of Jiangsu Province (WSW-019).

\section{References}

[1] E. C. Jauch, J. L. Saver, H. P. Adams et al., "Guidelines for the early management of patients with acute ischemic stroke: a guideline for healthcare professionals from the American Heart Association/American Stroke Association," Stroke, vol. 44, no. 3, pp. 870-947, 2013.

[2] W.-S. Juan, H.-W. Lin, Y.-H. Chen et al., "Optimal percoll concentration facilitates flow cytometric analysis for annexin V/propidium iodine-stained ischemic brain tissues," Cytometry Part A, vol. 81, no. 5, pp. 400-408, 2012.

[3] A. S. Go, D. Mozaffarian, V. L. Roger et al., "Heart disease and stroke statistics-2014 update: a report from the American heart association," Circulation, vol. 129, no. 3, pp. e28-e292, 2014.

[4] H. K. Eltzschig and T. Eckle, "Ischemia and reperfusion-from mechanism to translation," Nature Medicine, vol. 17, no. 11, pp. 1391-1401, 2011.

[5] C. Iadecola and J. Anrather, "The immunology of stroke: from mechanisms to translation," Nature Medicine, vol. 17, no. 7, pp. 796-808, 2011.

[6] A. Tuttolomondo, R. Di Sciacca, D. Di Raimondo, C. Renda, A. Pinto, and G. Licata, "Inflammation as a therapeutic target in acute ischemic stroke treatment," Current Topics in Medicinal Chemistry, vol. 9, no. 14, pp. 1240-1260, 2009.

[7] X. Kuang, L.-F. Wang, L. Yu et al., "Ligustilide ameliorates neuroinflammation and brain injury in focal cerebral ischemia/reperfusion rats: involvement of inhibition of TLR4/peroxiredoxin 6 signaling," Free Radical Biology \& Medicine, vol. 71, pp. 165-175, 2014.

[8] Y.-C. Wang, S. Lin, and Q.-W. Yang, "Toll-like receptors in cerebral ischemic inflammatory injury," Journal of Neuroinflammation, vol. 8, article 134, 2011.

[9] J. Cai and Y. Zhen, "Medicine in ancient China," in Medicine Across Cultures, pp. 49-73, Springer, 2003.

[10] J. Shang and X. J. Zhang, "Professor Zhang Shiqing's experience on treating pediatric allergic purpura with Xijiao Dihuang decoction," Journal of Gansu College of Traditional Chinese Medicine, vol. 3, pp. 6-7, 2005.

[11] B.-R. Zheng, J.-P. Shen, H.-F. Zhuang, S.-Y. Lin, Y.-P. Shen, and Y.-H. Zhou, "Treatment of severe aplastic anemia by immunosuppressor anti-lymphocyte globulin/anti-thymus globulin as the chief medicine in combination with Chinese drugs," Chinese Journal of Integrative Medicine, vol. 15, no. 2, pp. 145-148, 2009.

[12] Y.-R. Liu, P.-W. Li, J.-J. Suo et al., "Catalpol provides protective effects against cerebral ischaemia/reperfusion injury in gerbils," Journal of Pharmacy and Pharmacology, vol. 66, no. 9, pp. 12651270, 2014.

[13] N.-Y. Tang, C.-H. Liu, C.-T. Hsieh, and C.-L. Hsieh, “The anti-inflammatory effect of paeoniflorin on cerebral infarction induced by ischemia-reperfusion injury in sprague-dawley rats," American Journal of Chinese Medicine, vol. 38, no. 1, pp. 51-64, 2010.
[14] H. F. Lu, "Clinical study of Xijiao Dihuang decoction (Buffalo) for the treatment of acute cerebral hemorrhage," Jilin Journal of Traditional Chinese Medicine, vol. 28, pp. 718-719, 2008.

[15] W. Neuhaus, M. Burek, C. S. Djuzenova et al., "Addition of NMDA-receptor antagonist MK801 during oxygen/glucose deprivation moderately attenuates the upregulation of glucose uptake after subsequent reoxygenation in brain endothelial cells," Neuroscience Letters, vol. 506, no. 1, pp. 44-49, 2012.

[16] X. Qin, Z.-Q. Sun, X.-J. Dai et al., “Toll-like receptor 4 signaling is involved in PACAP-induced neuroprotection in BV2 microglial cells under OGD/reoxygenation," Neurological Research, vol. 34, no. 4, pp. 379-389, 2012.

[17] H. Luan, Z. Kan, Y. Xu, C. Lv, and W. Jiang, "Rosmarinic acid protects against experimental diabetes with cerebral ischemia: relation to inflammation response," Journal of Neuroinflammation, vol. 10, article 28, 2013.

[18] J.-M. Zhou, S.-S. Gu, W. H. Mei, J. Zhou, Z. Z. Wang, and W. Xiao, "Ginkgolides and bilobalide protect BV2 microglia cells against OGD/reoxygenation injury by inhibiting TLR2/4 signaling pathways," Cell Stress and Chaperones, vol. 21, no. 6, pp. 1037-1053, 2016.

[19] E. Hakimizadeh, M. K. Arababadi, A. Shamsizadeh, A. Roohbakhshd, and M. Allahtavakoli, "The possible role of toll-like receptor 4 in the pathology of stroke," Neuroimmunomodulation, vol. 23, no. 3, pp. 131-136, 2016.

[20] S.-C. Tang, T. V. Arumugam, X. Xu et al., "Pivotal role for neuronal Toll-like receptors in ischemic brain injury and functional deficits," Proceedings of the National Acadamy of Sciences of the United States of America, vol. 104, no. 34, pp. 13798-13803, 2007.

[21] J. Zaremba, P. Skrobanski, and J. Losy, “Tumour necrosis factoralpha is increased in the cerebrospinal fluid and serum of ischaemic stroke patients and correlates with the volume of evolving brain infarct," Biomedicine \& Pharmacotherapy, vol. 55, no. 5, pp. 258-263, 2001.

[22] J. R. Caso, J. M. Pradillo, O. Hurtado, J. C. Leza, M. A. Moro, and I. Lizasoain, "Toll-like receptor 4 is involved in subacute stress-induced neuroinflammation and in the worsening of experimental stroke," Stroke, vol. 39, no. 4, pp. 1314-1320, 2008.

[23] J. R. Caso, J. M. Pradillo, O. Hurtado, P. Lorenzo, M. A. Moro, and I. Lizasoain, "Toll-like receptor 4 is involved in brain damage and inflammation after experimental stroke," Circulation, vol. 115, no. 12, pp. 1599-1608, 2007.

[24] V. F. Belinga, G.-J. Wu, F.-L. Yan, and E. A. Limbenga, "Splenectomy following MCAO inhibits the TLR4-NF- $\kappa \mathrm{B}$ signaling pathway and protects the brain from neurodegeneration in rats," Journal of Neuroimmunology, vol. 293, pp. 105-113, 2016.

[25] C. Nito, H. Kamada, H. Endo, K. Niizuma, D. J. Myer, and P. H. Chan, "Role of the p38 mitogen-activated protein kinase/cytosolic phospholipase A2 signaling pathway in bloodbrain barrier disruption after focal cerebral ischemia and reperfusion," Journal of Cerebral Blood Flow \& Metabolism, vol. 28, no. 10, pp. 1686-1696, 2008.

[26] T. Borsellol, P. G. H. Clarkel, L. Hirt et al., "A peptide inhibitor of c-Jun N-terminal kinase protects against excitotoxicity and cerebral ischemia," Nature Medicine, vol. 9, no. 9, pp. 1180-1186, 2003.

[27] K. Ishitsuka, T. Ago, K. Arimura et al., "Neurotrophin production in brain pericytes during hypoxia: a role of pericytes for neuroprotection," Microvascular Research, vol. 83, no. 3, pp. 352-359, 2012. 
[28] S. S. Yu, J. Zhao, W. P. Zheng, and Y. Zhao, "Neuroprotective effect of 4-hydroxybenzyl alcohol against transient focal cerebral ischemia via anti-apoptosis in rats," Brain Research, vol. 1308, pp. 167-175, 2010.

[29] D. W. Nicholson and N. A. Thornberry, "Caspases: killer proteases," Trends in Biochemical Sciences, vol. 22, no. 8, pp. 299-306, 1997.

[30] M. R. Pulera, L. M. Adams, H. Liu et al., "Apoptosis in a neonatal rat model of cerebral hypoxia-ischemia," Stroke, vol. 29, no. 12, pp. 2622-2630, 1998. 


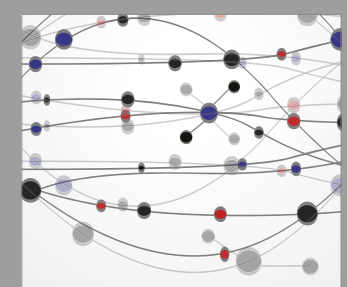

The Scientific World Journal
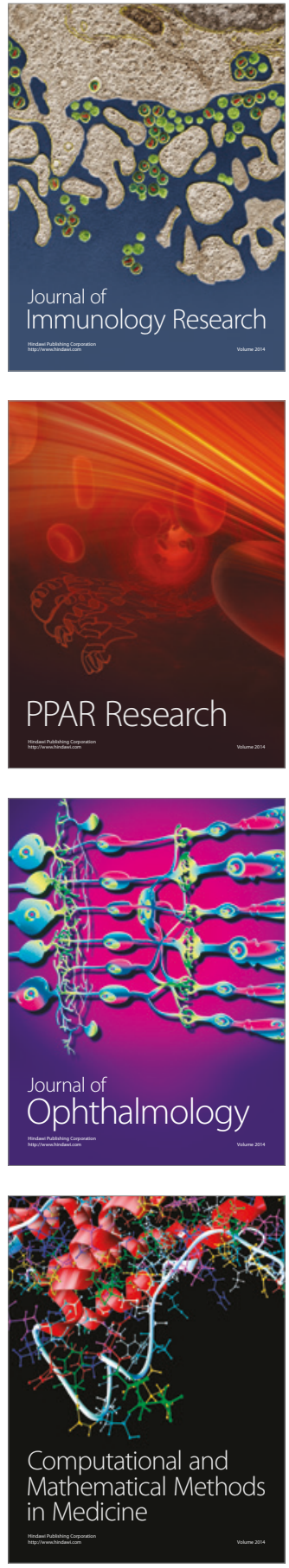

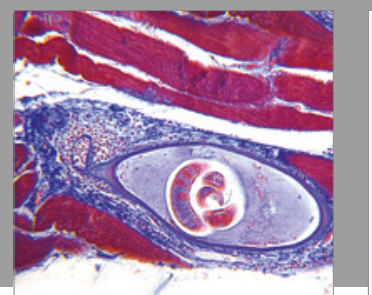

Gastroenterology Research and Practice
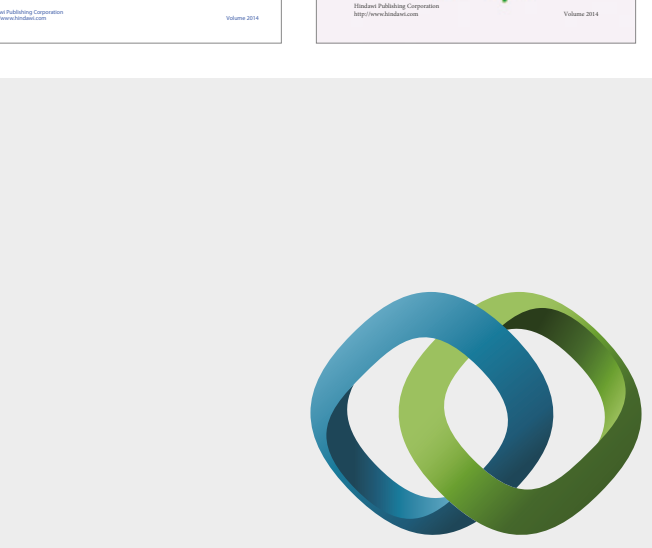

\section{Hindawi}

Submit your manuscripts at

https://www.hindawi.com
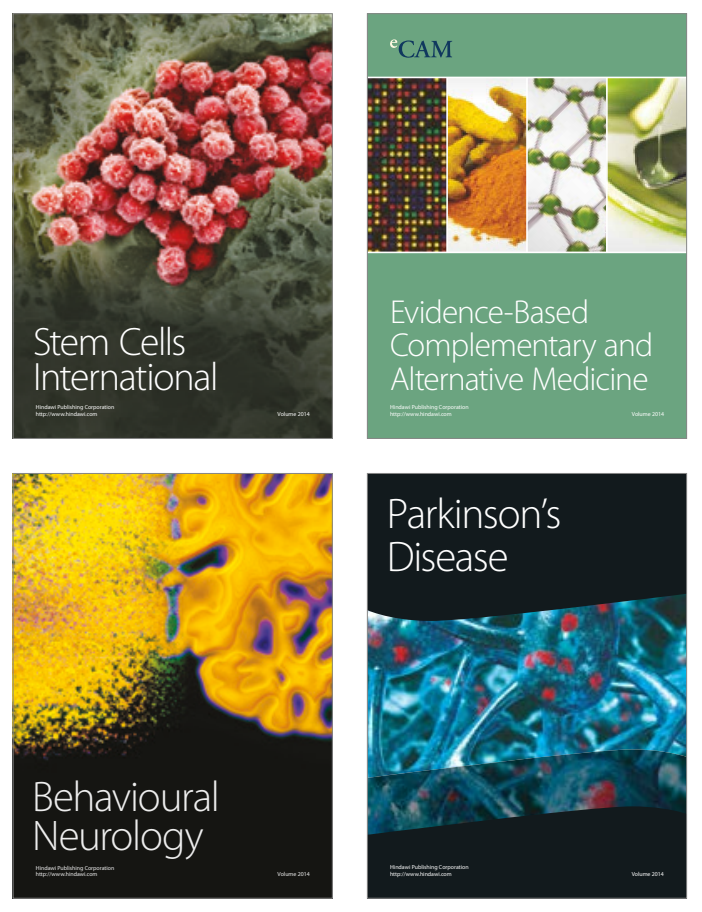
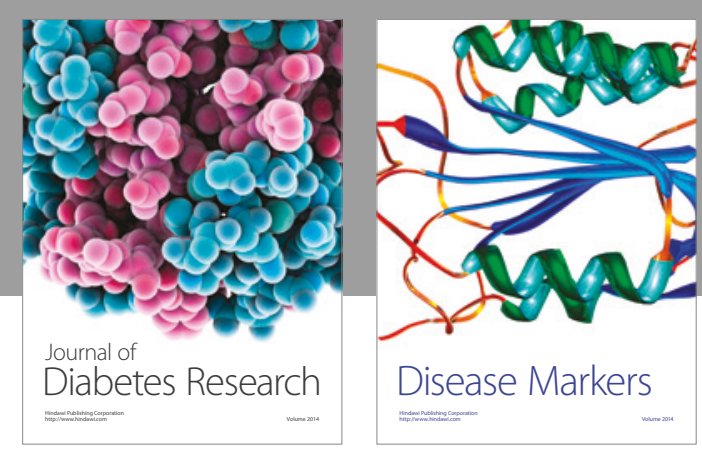

Disease Markers
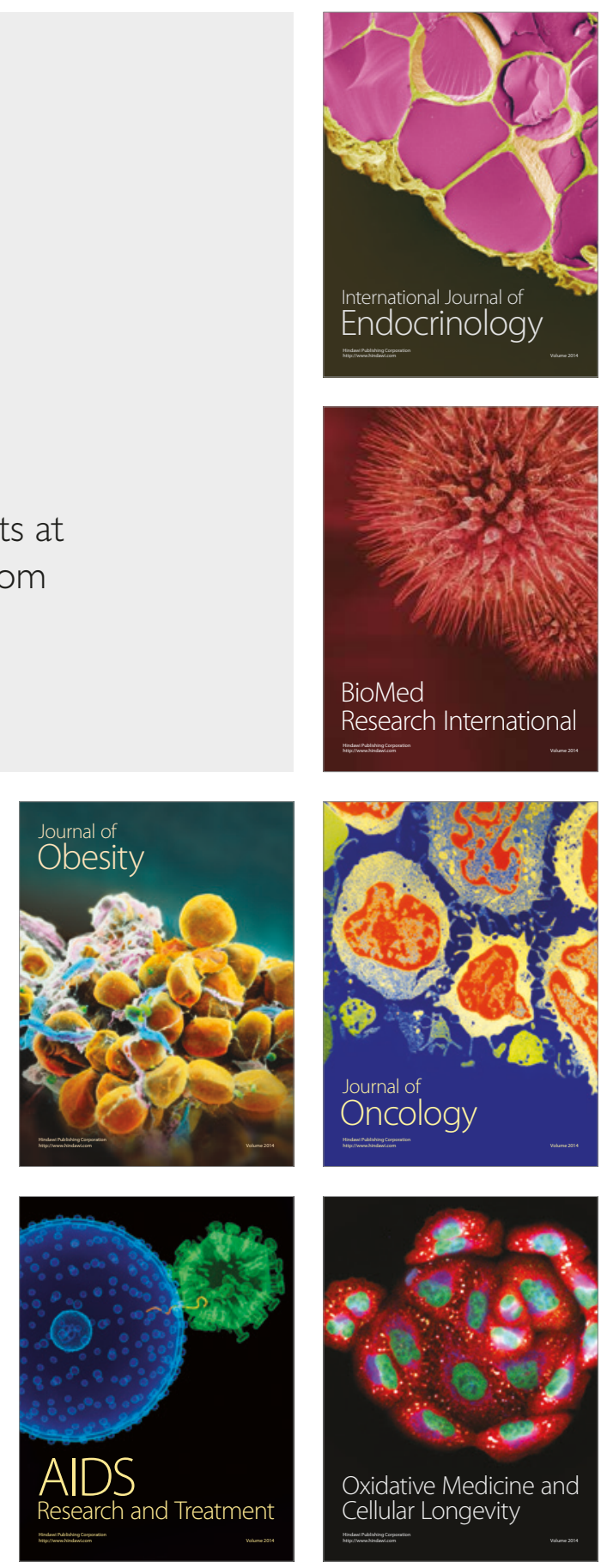